\title{
NOUVELLE
}

\section{Les glycosylations de PD-L1 sous les feux de la rampe}

Daniel Olive, Anne-Sophie Chrétien, Raynier Devillier,

Philippe Rochigneux, Laurent Gorvel, Jacques Nunès

Centre de Recherche en Cancérologie de Marseille (CRCM), Inserm UMR1068, CNRS UMR7258, Institut Paoli Calmettes, 27 boulevard Leï Roure, CS 30059, 13273 Marseille Cedex 09, France.

daniel.olive@inserm.fr
> Les anticorps monoclonaux antagonistes des molécules de cosignalisation négative de la réponse immunitaire, telles que CTLA-4 (cytotoxic T-lymphocyte-associated protein 4) et PD-1 (programmed cell death 1 ) ont révolutionné l'immunothérapie autologue contre de nombreux types de cancer, essentiellement des tumeurs solides. On connaît deux ligands de PD-1: PD-LI (programmed death-ligand 1) et PD-L2. La recherche actuelle porte principalement sur PD-Ll car il est exprimé par les cellules de nombreuses tumeurs et par des cellules du microenvironnement tumoral, sous l'influence de l'inflammation, de l'hypoxie, et des processus d'oncogenèse. Les questions concernant PD-Ll sont multiples. La plus pragmatique est «comment détecter l'expression de PD-Ll pour identifier les tumeurs les plus sensibles à l'immunothérapie?». En effet, l'expression de PD-Ll par les cellules tumorales (comme dans les cancers du poumon non à petites cellules), mais aussi par les cellules de l'immunité (comme dans les cancers du sein), constitue l'un des critères prédictifs de réponse à l'immunothérapie. Actuellement, la technique privilégiée pour les tumeurs solides, du fait de son utilisation par les anatomo-pathologistes en routine hospitalière, est l'analyse de l'expression de PD-Ll par immunohistochimie sur tissu inclus en paraffine. Cette approche - simple a priori - s'est cependant rapidement heurtée à la difficulté de disposer d'anticorps monoclonaux anti-PD-Ll utilisables en routine sur les fragments de tissus fixés après l'exérèse tumo- rale, ce qui a conduit à poser de nouvelles questions: Pourquoi l'obtention de ces anticorps monoclonaux est-elle si difficile? Peut-on trouver des stratégies permettant d'augmenter l'efficacité des techniques de détection de PD-Ll ? Ces questions sont importantes pour comprendre pourquoi certains patients dont la tumeur ou le micro-environnement tumoral semble ne pas exprimer PD-Ll, ou ne l'exprimer que faiblement, répondent pourtant à l'immunothérapie. Cela est-il dû à la faible sensibilité de la technique d'immunohistochimie ou à l'implication de PD-L2, pour la détection duquel les outils sont encore plus rares? L'article de Lee et al. vient d'apporter une réponse à cette question [1].

Les différents modes de contrôle, transcriptionnels et post-transcriptionnels, de l'expression du gène CD274 codant PD-Ll sont rappelés dans la Figure 1, adaptée de [2]. Parmi ceux-ci figure le nombre de copies de CD274 associé à l'amplification de la région chromosomique 9p24.1, par exemple dans les cellules de Reed-Sternberg caractéristiques des lymphomes de Hodgkin. Cette amplification se traduit par une augmentation du nombre de copies des gènes codant PD-L1, PD-L2 et JAK2 (Janus kinase 2), et de l'expression de ces gènes. Les délétions de la région 3' non-codante de I'ARNm codant PD-LI sont également associées à une augmentation de la quantité de ce transcrit. Parmi les mécanismes épigénétiques de contrôle de l'expression de CD274, on peut citer la méthylation du promoteur de ce gène, ainsi que le rôle des micro-ARN miR-
513, miR-570, miR-34s, et miR-200. La régulation transcriptionnelle implique les facteurs de transcription HIF, Myc, STAT, NFKB et $A P-1^{l}$, dont l'expression est elle-même régulée par des facteurs de croissance, des récepteurs à activité kinase, des interférons, l'hypoxie, ainsi que par l'augmentation de la concentration du lactate. Enfin, des mécanismes post-traductionnels impliquant phosphorylation, ubiquitination, et glycosylation contrôlent l'activité de PD-L1.

L'article de Lee et al. révèle le rôle des glycosylations. PD-Ll, comme la plupart des molécules de la superfamille des immunoglobulines, est fortement glycosylé. En effet, l'analyse par électrophorèse en gel de polyacrylamide montre que la forme non glycosylée de la protéine a une masse moléculaire d'environ $33 \mathrm{kDa}$, et qu'il existe des formes de masse moléculaire supérieure allant de 45 à $55 \mathrm{kDa}$. Cela suggère une importante hétérogénéité des molécules $\mathrm{PD}-\mathrm{Ll}$ présentes à la surface cellulaire. La digestion enzymatique des différentes formes de PD-Ll par des glycosidases indique qu'il s'agit de formes $\mathrm{N}$-glycosylées, par ajout de déterminants osidiques divers sur un résidu asparagine (N) dans un motif $\mathrm{N}-\mathrm{X}-\mathrm{T} / \mathrm{S}$ de la séquence protéique (où $T$ et $S$ désignent respectivement des résidus thréonine et sérine). Les résidus N35, N192, N200 et N219 de PD-L1 seraient susceptibles d'être glycosylés.

La glycosylation de PD-Ll était déjà connue comme un facteur important d'immuno-suppression [3-5]. En effet,

${ }^{1}$ hypoxia inducible factor; signal transducer and activator of transcription; nuclear factor-kappa B ; activating protein-1. 


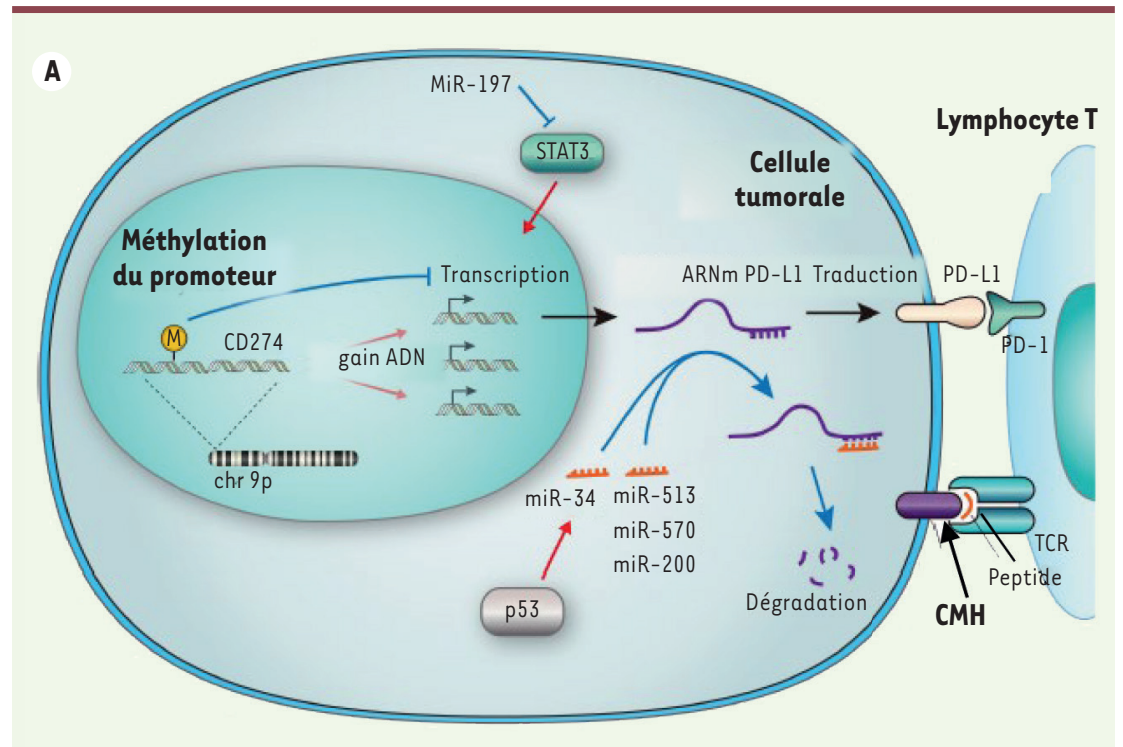

Figure 1. A. Contrôle génétique et épigénétique de l'expression de CD274 dans les cellules tumorales. Le gène CD274 codant PD-Ll est localisé dans une région du bras court du chromosome 9 (9p24.1) qui est amplifiée dans certains sous-types de cancers. L'augmentation du nombre de copies de CD274 augmente la quantité de ses ARNm, alors qu'une méthylation de ce gène inhibe sa transcription. Les microARN (miRNA) peuvent réguler la synthèse de PD-Ll en réprimant celle de STAT3 (signal transducer and activator of transcription 3), qui active CD274 en trans. Les miRNA peuvent également se lier à la région 3' non traduite de l'ARNm codant PD-LI, ce qui conduit à sa dégradation. L'expression du gène suppresseur de tumeur TP53 peut également réduire l'expression de CD274 via miR-34, un miRNA se liant à la région 3' non traduite de l'ARNm codant PD-Ll. L'interaction entre PD-Ll et $\mathrm{PD}-1$, concomitante à la présentation de l'anti-

B

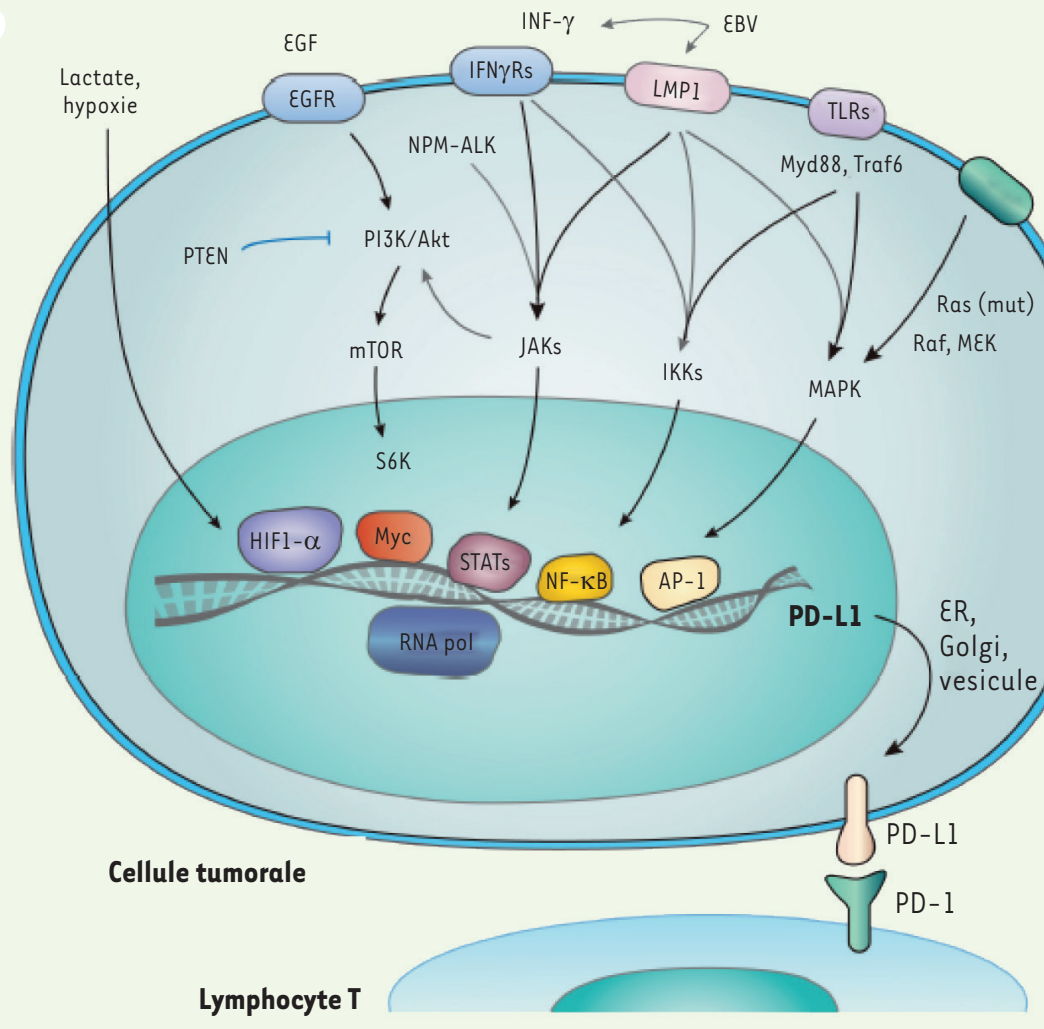

la glycosylation prévient la dégradation de PD-Ll par le protéasome et augmente la stabilité de la protéine d'un facteur quatre. Sa régulation est modifiée par les processus oncogéniques. Ainsi, la transition épithélio-mésenchyma- teuse induit l'expression de la sous-unité catalytiquement active de l'oligosaccharyl-transférase STT3 (dolichyl-diphosphooligosaccharide-protein glycosyltransferase subunit) dans les cellules souches tumorales [6], et la glycosylagène au TCR ( $T$-cell receptor) par le CMH (complexe majeur d'histocompatibilité), entraîne l'inhibition de la réponse anti-tumorale par les lymphocytes T. B. Activation transcriptionnelle de CD274 en réponse à différentes voies de signalisation. CD274 est transcrit en réponse à l'activation de diverses voies de signalisation, et donc de facteurs de transcription tels que HIF- $1 \alpha$, Myc, STAT, NF- $\kappa B$ et AP- 1 . Ces facteurs de transcription sont contrôlés par des voies de signalisation interconnectées impliquant EGF/ $\mathrm{PI3K} / \mathrm{AKT} / \mathrm{mTOR}$ (inhibé par PTEN), RTK/Ras/ Raf/MEK/ERK, IFN- $\gamma / J A K$ (également induites pas le gène muté NPM-ALK ainsi que LMPI activé par l'EBV), TLR/Myd88/Traf6/IKK, ainsi qu'un microenvironnement enrichi en lactate (figure adaptée de [2]). EGF : epidermal growth factor; PI3K : phosphatidyl-inositol 3-kinase; AKT : protéine kinase $B$; mTOR : mammalian target of rapamicyne; PTEN : phosphatase and tensin homolog; RTK : receptor tyrosine kinase ; MEK : MAPK/ERK kinase ; $\varepsilon R K$ : extracellularsignal-regulated kinase ; IFN-gamma : interferon gamma; JAK : Janus kinase ou Just another kinase; NPM-ALK : nucleophosmin-anaplastic lymphoma kinase; LMPI : latent membrane protein 1 ; EBV : Epstein-Barr virus ; TLR : Tolllike receptor; myD88: myeloid differentiation primary response 88; Traf6: TNF receptorassociated factor 6 ; IKK : inhibitor of nuclear factor kappa $B$. 


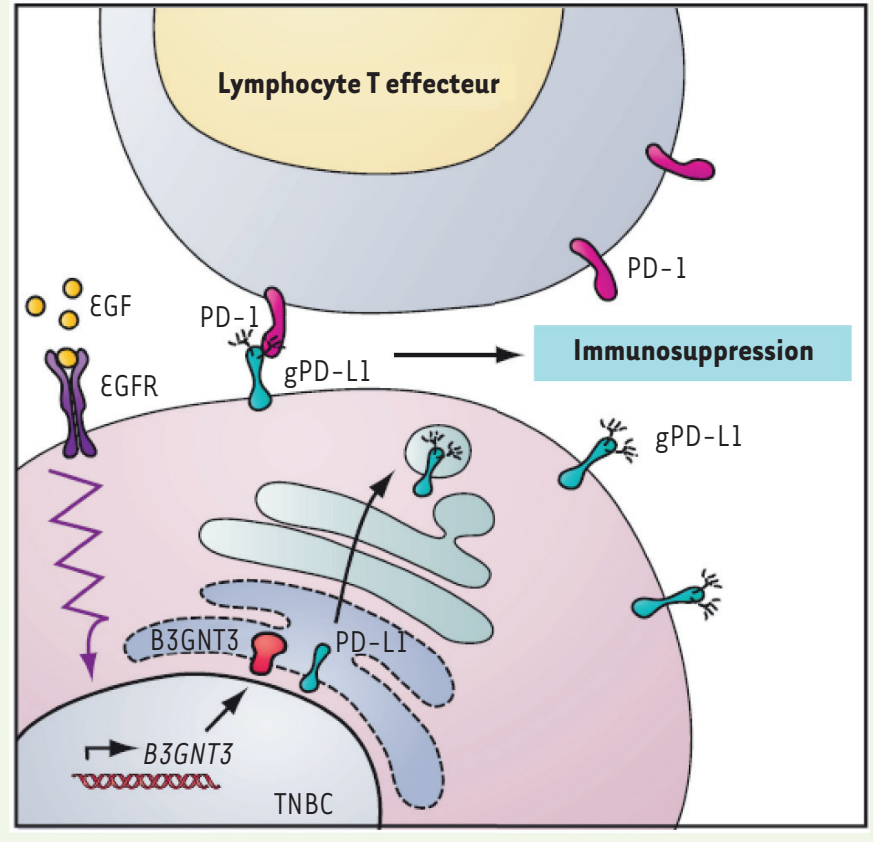

Figure 2. La glycosylation de PD-L1 contrôle l'immunosuppression tumorale dans les cancers du sein «triple négatif » (CSTN) (triple negative breast cancer, TNBC). Dans les CSTN, la glycosylation de PD-Ll est requise pour l'interaction avec PD-l et l'immunosuppression qui en résulte. La voie de signalisation EGF/EGFR augmente l'expression de la $\beta$-1,3-N-acétylglucosaminyltransférase B3GNT3, qui contrôle la $\mathrm{N}$-glycosylation de PD-L1, un processus essentiel pour la suppression de la réponse immunitaire (figure adaptée de [5]).

tion de PD-Ll qui en résulte est importante pour son interaction avec PD-1 et l'induction de l'immunosuppression: des souris mutantes chez lesquelles les résidus asparagine glycosylables de $\mathrm{PD}-\mathrm{Ll}$ ont été mutés présentent une activité immunosuppressive diminuée [3]. En plus de STT3, cette interaction entre PD- 1 et PD-Ll dépendrait également de la $\beta-1,3-\mathrm{N}$-acétylglucosaminyltransférase 3 (B3GNT3), qui induit la poly-N-acétyl-lactosamination (polyLacNac) de PD-Ll (Figure 2).

Lee et al. ont testé le rôle de la N-glycosylation dans la reconnaissance de PD-Ll par les anticorps monoclonaux dirigés contre la protéine. En faisant agir préalablement la peptide-N-glycosidase $F$ ( $P G$ Nase), qui clive les chaînes de $\mathrm{N}$-glycosylation, sur les coupes de tissu tumoral, ils ont doublé la sensibilité de la détection de PD-LI par immunohistochimie chez une par- tie des patients cancéreux traités par anticorps anti-PD-Ll. Ces résultats intéressants sont cependant à nuancer si l'on prend en considération l'expression de PD-Ll par les cellules immunitaires, qui est un critère d'inclusion pour l'immunothérapie chez les femmes atteintes de cancers du sein, et qui devra donc être testée de la même façon pour démontrer l'efficacité de cette technique. L'intérêt de cette approche - malgré tout imparfaite car ayant une sensibilité de détection encore limitée - est de proposer des stratégies de pré-traitement des échantillons histopathologiques qui augmenteraient la sensibilité de I'analyse immunohistochimique de PD-Ll avec les anticorps monoclonaux existants, sans préjuger des nouveaux anticorps monoclonaux actuellement en développement contre cette molécule. II reste à évaluer en pratique clinique, à travers les groupes collaboratifs d'anatomo-pathologistes au niveau international, la robustesse des résultats de cette étude pionnière. Les auteurs indiquent que ces observations seraient, entre autres, dues au fait que les anticorps monoclonaux utilisés jusqu'à présent pour détecter PD-Ll ont été obtenus par immunisation de souris contre des peptides pour la plupart synthétiques, donc non glycosylés. Cet argument est discutable dans la mesure ou la plupart des anticorps monoclonaux utilisés actuellement dans les analyses immunohistochimiques des échantillons tumoraux ont été obtenus à partir de fragments de la protéine PD-Ll produits dans des cellules eucaryotes. Il existe par ailleurs des anticorps monoclonaux dirigés spécifiquement contre les formes glycosylées de PD-Ll, et dont l'effet anti-tumoral dans des modèles précliniques est prometteur [4]. Une autre approche serait donc envisageable si ce sont les formes $\mathrm{N}$-glycosylées de PD-Ll qui sont les plus importantes pour la fonction immunosuppressive: l'enjeu serait alors de créer des anticorps monoclonaux spécifiques des formes glycosylées les plus immunosuppressives. Stratégie séduisante, qui implique cependant de montrer que les anticorps obtenus sont spécifiques des formes glycosylées de PD-Ll, et pas seulement de $\mathrm{N}$-glycosylations communes à un ensemble de protéines différentes détectables par immunohistochimie. De tels anticorps permettraient en théorie d'obtenir une efficacité de détection des patients susceptibles de répondre favorablement à l'immunothérapie dirigée contre PD-1 encore supérieure à celle obtenue par la déglycosylation préalable des coupes tissulaires, sous réserve de l'hétérogénéité de glycosylation entre les différentes tumeurs, entre les différentes cellules cancéreuses d'une même tumeur, ou encore entre les cellules cancéreuses et les cellules immunitaires du microenvironnement 
tumoral. Une première étape d'analyse de ces anticorps par immunohistofluorescence, en y associant des marqueurs des cellules tumorales et des cellules immunitaires, même sur échantillons de tissu congelés (qui ne sont pas utilisés en routine clinique, mais sont très utiles à l'étape de recherche), permettrait de valider cette stratégie. $\diamond$ PD-L1 glycosylation under the spotlights

\section{LIENS D'INTÉRÊT}

Les auteurs déclarent n'avoir aucun lien d'intérêt concernant les données publiées dans cet article.

\section{RÉFÉRENCES}

1. Lee HH, Wang YN, Xia W, et al. Removal of $\mathrm{N}$-linked glycosylation enhances PD-Ll detection and predicts anti-PD-1/PD-L1 therapeutic efficacy. Cancer Cell $2019 ; 36: 168-78$

2. Wang $\mathrm{Y}$, Wang $\mathrm{H}, \mathrm{YaO}_{\mathrm{O}} \mathrm{H}$, et al. Regulation of PD-LI: emerging routes for targeting tumor immune evasion. Front Pharmacol $2018 ; 9: 1-13$

3. Hsu JM, Li CW, Lai YJ, Hung MC. Posttranslational modifications of PD-Ll and their applications in cancer therapy. Cancer Res 2018 ;

$78: 6349-53$.

4. Li CW, Lim SO, Chung SM, et al. Eradication of triple-negative breast cancer cells by targeting glycosylated PD-L1. Cancer Cell 2018 33: 187-201.

5. Salatino M, Romina Girotti M, Rabinovich GA. Glycans pave the way for immunotherapy in triple-negative breast cancer. Cancer Cell 2018 ; 33 : 155-7.

6. Li CW, Lim SO, Chung EM, et al. ST3-dependent PD-L accumulation on cancer stem cells promotes immune evasion. Nat Com 2016; 7 : 12632 